\title{
Polymer Encapsulated Reverse Micelles: A Composite Material Design for the Optical Detection of Weak Magnetic Fields
}

C. Michael Elliott ${ }^{* *}$, Ulrich E. Steiner ${ }^{*}$,Joseph J. Kremer ${ }^{\dagger}$, and Karsten A. Hötzer ${ }^{*}$

\author{
Department of Chemistry, \\ Colorado State University, \\ Fort Collins, CO 80523 \\ And \\ Fachbereich Chemie, \\ Universität Konstanz, \\ Konstanz, Germany, D-78457
}

${ }^{\dagger}$ Colorado State University

¿niversität Konstanz 


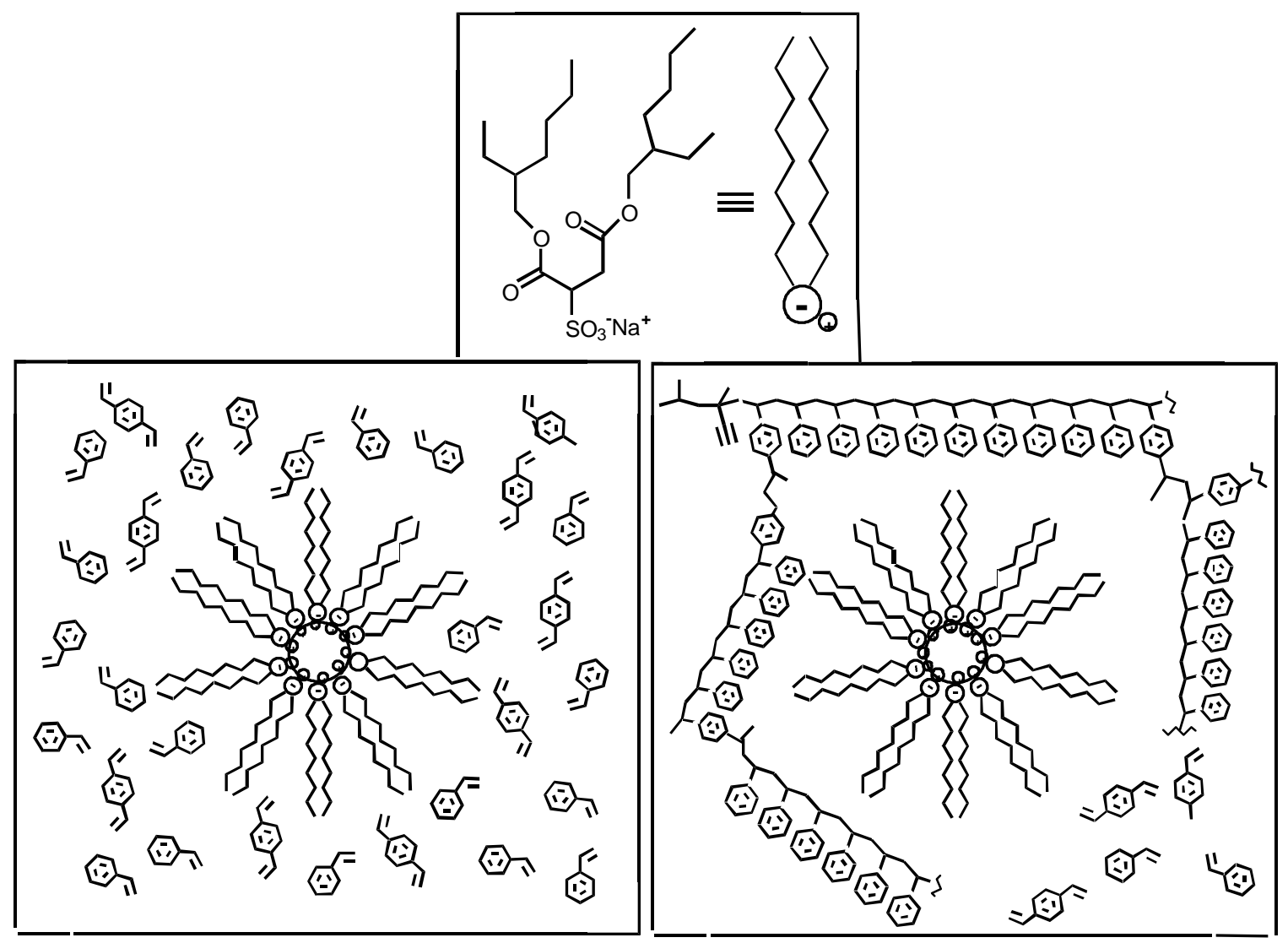

Supporting Scheme 1: Materials preparation of polymer encapsulated reverse micelle composites

Supporting Information: Explanation of triad magnetic field dependence.

Supporting Information

Hyperfine coupling of the nuclear magnetic moments to the unpaired electron spins provides a mechanism whereby the electron spins can lose their phase coherence (e.g., undergo triplet-singlet interconversion.) The efficiency of this spin interconversion is dependent upon the magnitude of the triplet-singlet energy difference relative to the hyperfine coupling energy. In the absence of any applied field, the singlet and triplet states of the CSS are essentially degenerate and the loss of spin coherence via this mechanism is rapid. When a magnetic field is present, the triplet experiences Zeeman splitting and the outer two components (i.e., $\mathrm{T}_{+}$and $\mathrm{T}_{-}$) are no longer degenerate with the singlet; thus, their rate of conversion to the singlet is slowed down. Consequently, in the presence fields as small as $5 \mathrm{mT}$, the average rate at which the CSS returns to the ground state changes dramatically. There is a fast component to the ground-state recovery that is field-independent $\left(\mathrm{T}_{0}\right)$ making up approximately onethird of the CSS population. The remaining two-thirds of the CSS population ( $\mathrm{T}_{+}$and $\left.\mathrm{T}_{-}\right)$return to the ground state with a rate that becomes progressively slower with increasing field. At ca. $500 \mathrm{mT}$, the 
process saturates and the rates of both the fast and slow components of ground-state recovery become constant as the field is further increased (up to at least $3000 \mathrm{mT}$ ). Phenomenologically, at the point where the field effect saturates, the average CSS lifetime in bulk solution has increased by ca. 10-fold (to over a microsecond) relative to the zero-field case. Simply holding a standard magnetic stir bar adjacent to the sample cuvette is sufficient to saturate the effect. Finally, because of large differences between CSS and ground-state spectra, the presence of MFs induces spectral changes that are straightforward to detect.

Materials: The experimental conditions for forming a specific PERM depends on the particular surfactant and polar phase employed. To produce AOT-based sensors, $0.5660 \mathrm{~g}$ of wet AOT (ca $0.46 \%$ $\mathrm{H}_{2} \mathrm{O}$ ) was added to a $25.00 \mathrm{~mL}$ volumetric flask which was then filled with the $2: 1$

styrene:divinylbenzene mixture. Two milliliter aliquots of this solution were dispensed into ( $45 \mathrm{x}$ 15) $\mathrm{mm}^{2} 4.0 \mathrm{~mL}$ Fisherbrand cylindrical screw-cap vials. In a dark room, using a Gastight 1710 glass microliter syringe, 9.0, 18.0, 27.0 and $36.0 \mu \mathrm{L}$, respectively, of $20.00 \mathrm{mM}$ triad solution in the polar solvent (either $\mathrm{H}_{2} \mathrm{O}$ or formamide) were injected into each of four vials containing the AOT solution. Sonication and mechanical vibration of the samples resulted in clear reverse micellar solutions with $\mathrm{W}_{\mathrm{o}}$ values ([polar phase]/[surfactant]) of 5.00, 10.00,15.00, or 20.00, respectively. The radical polymerization initiator, 2,z azobis[2,4-dimethylpentanenitrile] (ADPN), was dissolved in each reverse micellar solution $(1.9 \mathrm{mg}, 0.10$ weight percent). The vials were capped with serum caps and purged with nitrogen for several minutes to remove oxygen. A heating mantle filled with sand and set to $35^{\circ} \mathrm{C}$ was used to cure composites for 96 hours. The composites were retreived from their vials, ground to orthogonal dimensions amenable to spectroscopic measurements, and polished using 0.3 micron Buehler polish. 